\title{
Consumption effect of a synbiotic beverage made from soy and yacon extracts containing Bifidobacterium animalis ssp. lactis BB-12 on the intestinal polyamine concentrations in elderly individuals
}

\author{
Marla Simone Jovenasso Manzoni ${ }^{\mathrm{a}, *}$, Elizeu Antonio Rossi ${ }^{\mathrm{a}}$, Nadiége Dourado Pauly-Silveira ${ }^{\mathrm{a}}$, \\ Roseli Aparecida Pinto ${ }^{\mathrm{a}}$, Mariana Nougalli Roselino ${ }^{\mathrm{a}}$, Iracilda Zeppone Carlos ${ }^{\mathrm{b}}$, \\ Marcela Bassi Quilles ${ }^{b}$, Maria Beatriz de Abreu Glória ${ }^{c}$, Daniela Cardoso Umbelino Cavallini ${ }^{a}$ \\ a Departamento de Alimentos e Nutrição, Faculdade de Ciências Farmacêuticas, Universidade Estadual Paulista, Araraquara, SP, Brazil \\ b Departamento de Análises Clínicas, Faculdade de Ciências Farmacêuticas, Universidade Estadual Paulista, Araraquara, SP, Brazil \\ ${ }^{c}$ Departamento de Alimentos, Faculdade de Farmácia, Universidade Federal de Minas Gerais, Belo Horizonte, MG, Brazil
}

\section{A R T I C L E I N F O}

\section{Keywords:}

Soy

Yacon

Bifidobacterium

Polyamine

Gut environment

Elderly

\begin{abstract}
A B S T R A C T
This study aimed to investigate the effect of a synbiotic beverage made from soy and yacon (Smallanthus sonchifolius) extracts containing Bifidobacterium animalis ssp. lactis BB-12 on healthy elderly individuals' intestinal polyamine concentrations. A randomized, double-blinded, placebo-controlled trial has been conducted with twenty-nine volunteers (over 65 years of age) who either had a daily intake of $150 \mathrm{~mL}$ of synbiotic (synbiotic group - S) or placebo (placebo group - P) beverages. Both had the same nutrient composition, except that a probiotic culture was added to the synbiotic beverage. Total experiment time was 8 weeks, which was divided into 3 consecutive phases: a prefeeding period ( 2 weeks), followed by a feeding period ( 4 weeks) and a postfeeding period ( 2 weeks). Stool samples were collected at 3 time periods. Fecal concentrations of polyamines, putrescine (PUT), cadaverine (CAD) and spermidine (SPD) that were obtained during the synbiotic and placebo consumption period were significantly higher $(p<0.05)$ than those found during the pre-consumption baseline level period. No significant differences in the number of bifidobacteria, clostridia, or enterobacteria were observed in any of the two groups at the three time periods. Similarly, no significant effect on the production of proinflammatory cytokines tumor necrosis factor-alpha (TNF- $\alpha$ ), interleukin-6 (IL-6) and anti-inflammatory interleukin-10 (IL-10) was induced by the synbiotic or placebo beverages consumption. The results herein indicate that both the synbiotic and the placebo beverage consumption have increased polyamines levels, which are often reduced in elderly individuals, without influencing inflammatory responses. In addition, both placebo and synbiotic beverages seems to contribute by maintaining increased polyamines levels.
\end{abstract}

\section{Introduction}

The core microbiota of elderly individuals has been characterized by unusual phylum proportions and extreme variability (Claesson et al., 2011). Because of its crucial role in the host's physiology and healthy condition, age-related differences in gut microbiota composition may be related to diseases progression and a frail elderly population (Biagi et al., 2010). In this context, a growing understanding of the gut microbiota impact on human health has resulted in attempts to manipulate its composition by using probiotics and prebiotics, both from prophylactic and therapeutic perspectives (Biagi, Candela, FairweatherTait, Franceschi, \& Brigidi, 2012).

More recently, a study has demonstrated that the intake of arginine with the probiotic strain LKM512 can prevent aging-induced quality of life deterioration via polyamines upregulation (Kibe et al., 2014). Furthermore, it has been demonstrated that once intestinal polyamines concentrations were increased by LKM512 yogurt ingestion, it inhibited rat's senescence and enhanced their longevity, as well as inhibited elderly individuals' systemic inflammation, and reduced mutagenicity in healthy adults' guts (Matsumoto \& Benno, 2004; Matsumoto, Kurihara, Kibe, Ashida, \& Benno, 2011; Matsumoto, Ohishi, \& Benno, 2001).

As in plants, polyamines are also ubiquitous amongst mammalian cells, including human cells, which are involved in a wide range of vital cellular processes, playing essential roles in human development, metabolism and physiological functions, hence polyamines are essential to maintaining good health conditions at all life stages (Hunter \& Burrit,

\footnotetext{
* Corresponding author at: Rodovia Araraquara-Jaú, Km 1, Campus Universitário, Caixa Postal 502, CEP: 14802-901 Araraquara, São Paulo, Brazil.

E-mail address: cleber.manzoni@enalta.com (M.S.J. Manzoni).
} 
2012; Kantaria \& Gokani, 2011). Notwithstanding, there is a consensus in literature about a decrease in polyamine concentrations in the body due to aging, although this may be tissue-specific condition. The effect that it has on human health is still being investigated; however, enhanced polyamine intake appears to have a positive effect on human health, e.g. aging process, as opposed to immunosenescence and inflammaging (a chronic inflammatory status) (Binh, Soda, \& Kawakami, 2011; Hunter \& Burrit, 2012; Soda et al., 2009).

Polyamines, including putrescine, spermidine, and spermine, are amongst the most important bacterial metabolites in the intestine (Matsumoto, Sakamoto, \& Benno, 2009). In fact, it has been acknowledged presently that there are three sources of polyamines in humans: intracellular de novo synthesis of polyamines, dietary polyamines and polyamines produced as metabolites by the gut microbiota (Hunter \& Burrit, 2012).

Nowadays, it is well-established that $B$. animalis ssp. lactis BB-12 is a common commercial probiotic product that can be used in foods. Studies on BB-12 ingestion have shown that it offers many potential health benefits (Palaria, Johnson-Kanda, \& O'Sullivan, 2011). However, to the best of our knowledge, no study has reported the effect of the strain BB-12 ingestion on fecal production of polyamines.

Therefore, the aim of the present double-blind, randomized, controlled feeding trial was to study the effects of ingesting a new lowcalorie synbiotic beverage made from yacon (prebiotic source) and soy extracts, containing probiotic Bifidobacterium animalis ssp. lactis BB-12 (Manzoni, Cavallini, Pauly-Silveira, Roselino \& Rossi, 2012) on elderly individuals' fecal polyamines concentration. It was found relevant to ascertain whether the synbiotic beverage consumption has also had an effect on stimulated cytokine production. In addition, intestinal bifidobacteria, clostridial, and enterobacterial numbers were determined.

\section{Materials and methods}

\subsection{Synbiotic and placebo beverages production}

The synbiotic and placebo beverages were processed at UNISOJA (Development and Production Unit for Soybean Derivates) in the Food Nutrition Department of the School of Pharmaceutical Sciences, UNESP, Araraquara (SP, Brazil). Procedures for preparing the beverages were described in detail by our previous report (Manzoni et al., 2012). They had the same nutrient composition, except that a probiotic culture was added to the synbiotic beverage (BB-12 ${ }^{\circledR}$-Probiotic-culture-Probio-Tec ${ }^{\circledR}$, Christian Hansen, São Paulo, Brazil). Yacon and soy extracts were used as the raw material to produce the placebo and the synbiotic beverage. The chemical composition of the beverages is presented in Table 1 . Microbiological analyses of the synbiotic product showed that the beverage exhibited Bifidobacterium ssp. counts of $10^{10} \mathrm{CFU}$ per $100 \mathrm{~mL}$ of product (Manzoni et al., 2012). $150 \mathrm{~mL}$ of the synbiotic and placebo beverage were packaged in appropriate polypropylene plastic food containers. The products were stored under refrigeration $\left(4 \pm 1{ }^{\circ} \mathrm{C}\right)$

Table 1

Chemical composition of the synbiotic and placebo beverages. ${ }^{\text {a }}$

\begin{tabular}{ll}
\hline Component & $(\mathrm{g} / 100 \mathrm{~g})$ \\
\hline Moisture & $92.96 \pm 0.01$ \\
Total solids & $7.25 \pm 0.01$ \\
Protein & $2.91 \pm 0.17$ \\
Fat & $1.42 \pm 0.01$ \\
Ash & $0.30 \pm 0.03$ \\
Total carbohydrate & $2.41 \pm 0.07$ \\
FOS content & $0.82 \pm 0.00$ \\
Energy value $(\mathrm{kJ} / 100 \mathrm{~g})$ & 148.22 \\
\hline
\end{tabular}

${ }^{a}$ Manzoni, Cavallini, Pauly-Silveira, Roselino, and Rossi (2012). Note. Synbiotic and placebo beverages had the same nutrient composition, except that a probiotic culture (Bifidobacterium animalis ssp. lactis BB-12) was added to the synbiotic beverage. until consumption. The products were produced weekly and freshly delivered to each volunteer.

\subsection{Participants}

The study protocol and consent procedures were approved by the Ethical Committee of the School of Pharmaceutical Sciences at the São Paulo State University (Process number 37/2007). Before conducting this study, written informed consents forms were filled out by all volunteers. Participants were recruited from the Open University of the Elderly (São Carlos, São Paulo, Brazil). A total of forty healthy elderly volunteers have been selected after physical examination, but only twenty-nine of them completed the study (fifteen in the placebo group and fourteen in the synbiotic group). The average age of the volunteers in the placebo group was 71 years old, while that of the volunteers in the synbiotic group was 67 . They have no antibiotic treatment for 3 months prior to the study period and no chronic or acute diseases. Volunteers were advised to maintain their normal life style, but were requested to refrain from consuming foods with probiotics. It is important to mention that there was no report on adverse events. The withdrawals in the synbiotic and placebo groups, six and five, respectively, were due to pneumonia with subsequent antibiotic treatment (one), and/or personal reasons.

\subsection{Experimental design}

This is a double-blind study in which the volunteers were randomly divided into two groups that either received the synbiotic (synbiotic group $-S$ ) or placebo beverages (placebo group $-\mathrm{P}$ ). Total experiment time was 8 weeks and was divided into 3 consecutive phases: a prefeeding period ( 2 weeks), followed by a feeding period ( 4 weeks) and a postfeeding period ( 2 weeks). Three stool samples were collected: one during the initial period (week 1), another one at the end of the ingestion period (week 6) and one at the end of the wash out period (week 8), which were stored at $-80{ }^{\circ} \mathrm{C}$ until analyses.

\subsection{Fecal polyamines concentration determination}

For extracting the polyamines from the each stool sample, frozen feces were 7 -fold diluted in 5\% trichloroacetic acid (TCA). The samples had been shaken for $10 \mathrm{~min}$ by using a vortex mixer, which were centrifuged at $10,000 \times g$ for $10 \mathrm{~min}$ at $4^{\circ} \mathrm{C}$ after homogenization. The supernatant was collected and the sediment was extracted twice with $7.0 \mathrm{~mL}$ and $6.0 \mathrm{~mL}$ of $5 \%$ TCA, respectively. The combined supernatant was filtrated in a millipore membrane mesh $(0.45 \mu \mathrm{m})$. The amines (putrescine, cadaverine, spermidine, and spermine) were separated by ion-pair reversed-phase HPLC and quantified fluorimetrically after postcolumn derivation with o-phthalaldehyde (Cirilo et al., 2003). The liquid chromatography was performed on a LC-10AD system connected to a RF-551 spectrofluorimetric detector at 340 and $445 \mathrm{~nm}$ of excitation and emission, respectively, and a CBM-10AD controller (Shimadzu, Kioto, Japan). A $300 \times 3.9 \mathrm{~mm}$ i.d. and $10 \mu \mathrm{m}$ reversed-phase $\mu$ Bondapaq C18 column, was used with a $\mu$ Bondapaq C18 guard-pack insert (Waters, Milford, MA, USA). The mobile phases were: A, solution of $0.2 \mathrm{M}$ sodium acetate, and $15 \mathrm{mM}$ 1-octanesulfonic acid sodium salt adjusted to $\mathrm{pH} 4.9$ with glacial acetic acid; and B, acetonitrile. The flow rate was set at $0.8 \mathrm{~mL} / \mathrm{min}$ and the gradient was: $13 \mathrm{~min}$ at $11 \% \mathrm{~B}$, $19 \mathrm{~min}$ at $30 \% \mathrm{~B} ; 24 \mathrm{~min}$ at $11 \% \mathrm{~B}$, and $45 \mathrm{~min}$ at $11 \% \mathrm{~B}$. The postcolumn derivatization reagent was delivered at $0.4 \mathrm{~mL} / \mathrm{min}$. It consisted of $1.5 \mathrm{~mL}$ Brij-35, $1.5 \mathrm{~mL}$ mercaptoethanol and $0.2 \mathrm{~g}$ o-phthalaldehyde dissolved in a $500 \mathrm{~mL}$ solution of $25 \mathrm{~g}$ boric acid and $22 \mathrm{~g}$ $\mathrm{KOH}$ (pH adjusted to 10.5 with $3 \% \mathrm{KOH}$ ). The column and the postcolumn reaction apparatus were at room temperature $\left(22 \pm 1{ }^{\circ} \mathrm{C}\right)$. The amines were identified by a comparison of their retention time in samples with standard solutions and also by adding the suspected amine to the sample. Amine levels were calculated by direct 
A

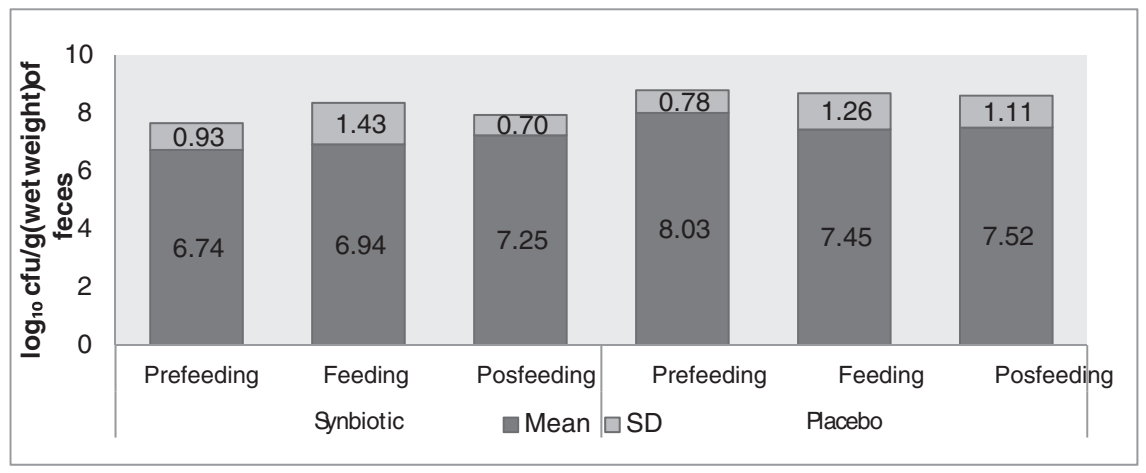

B

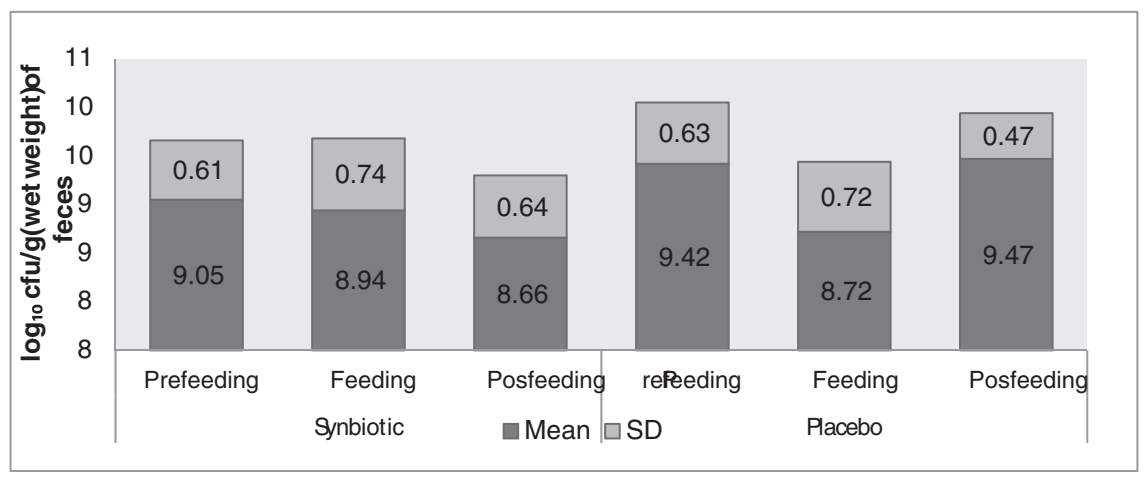

C

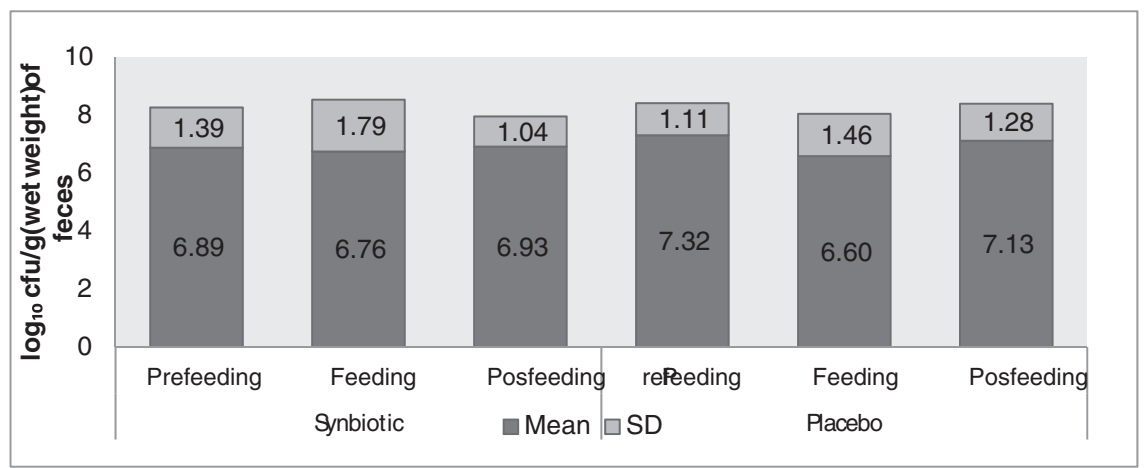

Fig. 1. Influence of synbiotic and placebo consumption in the fecal microbial counts of different groups of bacteria. Results are expressed as mean $\log _{10} \mathrm{cfu} / \mathrm{g}$ (wet weight) of feces \pm standard deviation ( $n=6$ /group). $p>0.05$. Note. The prefeeding period was week 1 , the feeding period was week 6 , and the postfeeding period was week 8. (A) Bifidobacterium spp. (B) Clostridium spp. (C) Enterobacteria. interpolation in the standard curve $(R \geq 0.9926)$. The detection limit of each polyamine was $0.02 \mathrm{mg} / 100 \mathrm{~g}$. Bioactive amine standards were purchased from Sigma Chemical Co. (St. Louis, MO, EUA). They included putrescine dihydrochloride, spermidine trihydrochloride, spermine tetrahydrochloride and cadaverine dihydrochloride.

\subsection{Enumeration of fecal bacteria assessment}

Microorganism analyses were conducted with feces from six volunteers of each group, which were collected in all set periods. Stool samples ( $1 \mathrm{~g}$ ) were serially 10 -fold diluted in sterile peptone water $(99 \mathrm{~mL})$, spread onto selective agar plates and then incubated under anaerobic conditions (Probac ${ }^{\oplus}$, São Paulo, Brazil). Bifidobacteria counts were obtained by using Bifidobacteria Iodoacetate Medium-25 (BIM25), according to the method described by Munoa and Pares (1988). Clostridium and enterobacteriaceae levels were enumerated by using Reinforced Clostridial Agar $\left(48 \mathrm{~h}, 37^{\circ} \mathrm{C}\right)$ and MacConkey medium $\left(24 \mathrm{~h}, 37^{\circ} \mathrm{C}\right)$, respectively. Samples were inoculated in duplicate in all media used in this study. The number of bacteria was presented as log
$\mathrm{CFU} / \mathrm{g}$ of wet weight of feces.

\subsection{Measurement of TNF- $\alpha$, and cytokines levels}

The feces of all volunteers (twenty-nine) at the three times periods were used for investigating the macrophage-derived cytokine production level. For such a purpose, a murine macrophage-like cell line, RW364, was co-cultured with fecal extracts from elderly volunteers. Fecal extracts were prepared as described by Matsumoto and Benno (2006).

\subsubsection{Cell line}

The murine macrophage-like cell line RW364, was kindly provided by the Pathology Department of the School of Odontology, UNESP, Araraquara (SP, Brazil). The cells were maintained in culture flasks (Corning Inc., USA) containing D-MEM medium (41,500; Life Technologies, Inc.), supplemented with $5 \%$ fetal bovine serum, $2 \mathrm{mM} \mathrm{L}_{\mathrm{L}}$ glutamine and $80 \mu \mathrm{g} \mathrm{mL}^{-1}$ gentamicin at $37{ }^{\circ} \mathrm{C}$ in an $7.5 \% \mathrm{CO}_{2}$ atmosphere. All chemicals were purchased from Sigma-Aldrich (St. Louis, 
MO, USA).

\subsubsection{IL-6, IL-10 and TNF- $\alpha$ production}

Cells were suspended in a RPMI-1640-C medium (Invitrogen Corp.,

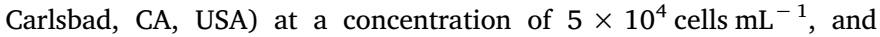
$990 \mu \mathrm{L}$ of the cell suspension was placed onto a 24 -well flat-bottom plate (Corning, Inc.). The cells were incubated for $48 \mathrm{~h}$ with lipopolysaccharide (LPS) of E. coli; $10 \mu \mathrm{L}$ LPS at a concentration of $1 \mu \mathrm{g} \mathrm{mL}^{-1}$ plate-adhering macrophage, and $20 \mu \mathrm{L}$ of the fecal extract at $37^{\circ} \mathrm{C}$ in $7.5 \% \mathrm{CO}_{2}$. After incubation, the samples were centrifuged and the supernatants were collected and stored at $-80{ }^{\circ} \mathrm{C}$ until assayed.

Tumor necrosis factor (TNF)- $\alpha$ and cytokine quantification in the supernatant culture were analyzed by using commercially available sandwich enzyme linked immunoassay (ELISA) kits (Ready SET GO! Elisa Sets ${ }^{\circledR}$, and Bioscience) according to manufacturer's instructions.

ELISA measurements were performed in triplicates. Results were expressed in $\mathrm{pg} / \mathrm{mL}$.

\subsection{Statistical analysis}

Data are reported as mean \pm standard deviations. The analysis of variance (ANOVA) was performed. Tukey's post hoc analysis was performed to determine the means that were different from one another. Differences $<0.05$ were considered significant.

\section{Results and discussion}

\subsection{Enumeration of fecal bacteria}

In this study, it has been found that the consumption of a synbiotic beverage made from soy and yacon extracts containing $B$. animalis ssp. lactis BB-12 has not induced a rise in total bifidobacteria levels as measured by plating. Actually, as shown in Fig. 1A, the mean of fecal bifidobacteria counts in the synbiotic and placebo groups were similar and remained unchanged during the different periods of the study (prefeeding, feeding, and postfeeding). Additionally, the genus Bifidobacterium was detected in all stool samples. In contrasting, in a randomized controlled study, intervention with a fermented oat drink containing a commercial $B$. animalis ssp. lactis BB-12 resulted in increased levels of $B$. animalis, however no significant changes in other bifidobacteria levels were observed (Ouwehand et al., 2008). This may be due to different methodologies being applied. In the present study, a cultured-based method has been used, while Ouwehand et al. (2008) relied on molecular methods. The authors pointed out that the presence of $B$. animalis might have been overlooked in earlier culture-based studies, as $B$. animalis may grow slowly on nutrient media that are usually used for culturing Bifidobacterium, and that it may be difficult to detect minor components of complex microbiota due to dominant species overgrowth (Lahtinen et al., 2009; Ouwehand et al., 2008). The discrepancies in Bifidobacterium detection may be explained considering the studies duration as well. A 6-month consumption of probiotic products resulted in modest increases in Bifidobacterium species levels (Ouwehand et al., 2008), while no changes were reported by following a 4-week synbiotic supplementation in the present study. This is consistent with another study that had evaluated the effects of a yogurt supplemented with inulin and the BB-12 strain on human intestinal bifidobacterial numbers by using a probe based on a real-time PCR approach, in which it was also pointed out that there were no significant differences in bifidobacteria numbers after a 4-week synbiotic consumption period (Palaria et al., 2011). However, it is also conceivable that the presence of Bifidobacterium at high levels and in all stool samples of elderly volunteers, which has been found herein, are in agreement with a previous study conducted by Lahtinen et al. (2009). In our study, it seemed as though the BIM-25 medium could not be used as a selective medium for isolating the Bifidobacterium animalis, but it could have be used successfully as a selective medium for
Bifidobacterium spp. isolation, given that, in this study, the fecal Bifidobacterium level was high in elderly subjects at the baseline level, which is consistent with recent reports from studies using molecular methods (Palaria et al, 2011; Ouwehand et al., 2008; Lahtinen et al., 2009; Ouwehand, Tiihonen, Saarinen, Puttala, \& Rautonen, 2009; Bartosch, Fite, Macfarlane, \& McMurdo, 2004). In a recent description of the Human Intestinal Tract Chip (HITChip) technology, the authors concluded that microbial composition and the gut microbiota diversity of young adults and seventy-years old individuals is highly similar, but it differs significantly from that of centenarians (Biagi et al., 2010). Based on the obtained results, they suggested that the threshold for an aged microbiota should be raised to 75-80 years of age. Life style has been shown to be effective at maintaining fecal bifidobacterial populations in clinical trials. In this context, an investigation has been conducted to monitor bacterial groups in stool samples obtained from older people, thus reporting that elderly people that still had relatively high levels of fecal bifidobacteria were very cognizant and lively (Bartosch et al., 2004). On the other hand, reductions in bifidobacteria levels were found in hospitalized elderly patients, either receiving antibiotic treatment or not, which is another point yet to be considered, as it had been reported earlier. According to the authors, the synbiotic beverage (containing Bifidobacterium bifidum, Bifidobacterium lactis, and oligofrutose) consumption by elderly people has significantly increased the counts of this species, which was particularly evident in individuals who had, at the beginning of the study, little or no B. bifidum in their fecal samples, whereas in volunteers with high initial counts of this organism, no increase was detectable (Bartosch, Woodmansey, Paterson, McMurdo, \& Macfarlane, 2005). Nevertheless, another research has pointed out that when initial bifidobacterial numbers are already high, it is difficult to further increase the size of the population by ingesting exogenous bifidobacterial cells (Alander et al., 2001). More recently, other authors supported the idea that the ingestion of the synbiotic yogurt may modulate total bifidobacterial numbers in people with either below-average levels of bifidobacteria or aboveaverage levels of clostridia (Palaria et al., 2011). No significant differences in numbers of clostridia, or enterobacteria were observed between the synbiotic and placebo groups during any of the feeding periods (Fig. 1B and $\mathrm{C}$, respectively). The results indicate that the synbiotic beverage ingestion does not statistically modulate enterobacterial and clostridial numbers in elderly people, which is in agreement with a previous study (Palaria et al, 2011). These findings differ from another study using BB-12, which did find a reduction in cell numbers of Enterobacteriaceae and Clostridium ssp. However, it involved a subject group of preterm infants (Mohan et al., 2006).

In the present study, the ingestion of FOS from yacon with an average daily dose of $1.2 \mathrm{~g} / 150 \mathrm{~mL}$ of synbiotic drink had no significant effect on fecal BB-12 numbers. Researchers have accepted that log increases in bifidobacteria counts do not necessarily correlate with administered daily doses, but it rather depends more on the initial bifidobacteria numbers (Reyed, 2007; Roberfroid, 2005). A previous study reported that lower initial bifidobacteria numbers have been shown to generate greater increases, regardless of dosage, within a range of 4-20 g or more per day (Roberfroid, 1998). Therefore, it can be speculated that, in this study, no increases were detectable which was possibly due to volunteers having high initial counts of this bifidobacteria. It is important to mention that the microbial counts presented $\left(10^{10} \mathrm{CFU}\right.$ per $100 \mathrm{~mL}$ of product) suggesting that beverage made from yacon, a vegetal prebiotic source, and soy extracts can be considered an adequate food matrix for supplementation by probiotic bacteria. In fact, the food matrix has been a topic of current research in the probiotic field (Lollo, Morato, Moura, Oliveira et al., 2015; Lollo et al., 2013).

\subsection{Fecal polyamines, TNF- $\alpha$, and cytokine levels}

Fecal polyamines concentrations in elderly volunteers are shown in Table 2. With the exception of spermine, during the feeding period, 
Table 2

Effect of the synbiotic and placebo ingestion on fecal polyamines concentrations in elderly people.

\begin{tabular}{llll}
\hline $\begin{array}{l}\text { Concentrations } \\
\left(\mathrm{mg} 100 \mathrm{~g}^{-1}\right)\end{array}$ & Prefeeding period & Feeding period & Posfeeding period \\
\hline PUT & & & \\
Synbiotic & $0.41 \pm 0.01$ & $0.71 \pm 0.01^{*}$ & $0.37 \pm 0.02^{* \#}$ \\
Placebo & $0.02 \pm 0.00$ & $0.13 \pm 0.02^{*}$ & $0.50 \pm 0.01^{* \#}$ \\
CAD & & & \\
Synbiotic & $6.41 \pm 1.24$ & $38.75 \pm 4.70^{*}$ & $41.97 \pm 6.95^{*}$ \\
Placebo & $0.02 \pm 0.00$ & $9.58 \pm 0.06^{*}$ & $4.63 \pm 0.30^{* \#}$ \\
SPD & & & \\
Synbiotic & $8.04 \pm 0.06$ & $11.13 \pm 0.35^{*}$ & $10.84 \pm 0.16^{*}$ \\
Placebo & $4.75 \pm 0.15$ & $7.09 \pm 0.29 *$ & $6.63 \pm 0.28^{* \#}$ \\
SPM & & & \\
Synbiotic & $1.44 \pm 0.54$ & $1.31 \pm 0.30$ & $1.45 \pm 0.53$ \\
Placebo & $1.86 \pm 0.98$ & $1.44 \pm 0.54$ & $1.60 \pm 0.65$ \\
\hline
\end{tabular}

Results are expressed as mean $\pm \mathrm{SD}(n=14$ for synbiotic group and, $n=15$ for placebo group). ${ }^{*} p<0.05$ vs prefeeding period; ${ }^{*} p<0.05$ vs feeding period. PUT, putrescine; CAD, cadaverine; SPD, spermidine; SPM, spermine. Note. The prefeeding period was week 1 , the feeding period was week 6 , and the postfeeding period was week 8 .

fecal concentrations of bioactive amines were significantly higher in the synbiotic and placebo group in comparison with the prefeeding period. Based on these results, soy extract in the composition of the beverages $(60 \%)$ seems to contribute as source of polyamines, once synbiotic and placebo beverages had the same nutrient composition, except that, a probiotic culture was added to the synbiotic beverage. A study with a Brazilian culture has confirmed soybean as a rich source of amines (Glória, Tavares-Neto, \& Labanca, 2005). On the other hand, researchers affirm that the proportion of polyamines that may be available for the metabolic demand of large intestinal mucosal tissues will be primarily from microbial origin rather than a dietary origin (Noack, Kleessen, Proll, Dongowaki, \& Blaut, 1998). Later on, another research has been conducted by the same authors who concluded that colonic microbiota is able to produce large amounts of polyamines in vivo (Noack, Dongowski, Hartmann, \& Blaut, 2000). Recently, evidence has been provided that the polyamine content in the gut is highly influenced by the metabolic activity of intestinal bacteria. In vitro studies have shown for the capacity of various strains of Bifidobacterium to produce polyamines. The microbial types were specially favored by FOS consumption and this fact could explain the effect of the FOS to change the cecal polyamines levels (Sabater-Molina et al., 2011). In agreement with this, studies have found that the probiotic strain Bifidobacterium animalis subsp. lactis LKM512 is able to increase intestinal luminal polyamines concentrations (Matsumoto \& Benno, 2004; Matsumoto \& Benno, 2006; Matsumoto et al., 2001; Matsumoto et al., 2009; Matsumoto et al., 2011). However, it was clear that the ingestion of LKM512 per se is not sufficient for elevating intestinal polyamines concentrations. Moreover environmental components can modulate polyamines absorption and release intestinal bacteria (Kibe et al., 2014; Matsumoto et al., 2011).

In the present study, fecal concentrations of CAD and SPD remained significantly elevated (compared to the feeding period), even when synbiotic beverage administration had ceased. The results of a previous study (Matsumoto et al., 2001) showed that PUT and SPM increased slightly when the probiotic yogurt administration had ceased. Authors argue that the decrease in bacteria that absorbed polyamines might lead to an increasing number of polyamines in the intestinal tract. On the other hand, a significant increase in PUT, CAD and SPD polyamines concentrations for placebo group in the final washout period (postfeeding period) was very evident compared to the prefeeding period. Clearly, further studies are required to verify the relationships that either the probiotic microorganism and/or the prebiotics present in soy and yacon affected fecal polyamines in the present study.
Table 3

Effects of the fecal extracts on stimulated in vitro production of cytokines before, during and after daily synbiotic and placebo intake.

\begin{tabular}{llll}
\hline $\begin{array}{l}\text { Concentration } \\
\left(\mathrm{pg} \mathrm{mL}^{-1}\right)\end{array}$ & Prefeeding period & Feeding period & Posfeeding period \\
\hline $\begin{array}{l}\text { IL-6 } \\
\text { Synbiotic }\end{array}$ & $568.89 \pm 147.06$ & $789.53 \pm 123.02$ & $518.32 \pm 157.98$ \\
Placebo & $573.24 \pm 150.37$ & $784.89 \pm 187.07$ & $564.38 \pm 220.35$ \\
IL-10 & & & \\
Synbiotic & $114.33 \pm 45.67$ & $141.00 \pm 4.24$ & $121.42 \pm 44.87$ \\
Placebo & $77.25 \pm 52.04$ & $71.89 \pm 5.63$ & $56.47 \pm 11.41$ \\
TNF- $\alpha$ & & $1189.10 \pm 343.69$ & $1141.30 \pm 397.68$ \\
Synbiotic & $1239.50 \pm 291.18$ & $1341.70 \pm 119.91$ & $1022.00 \pm 169.02$ \\
Placebo & $1220.50 \pm 254.91$ & 130.04 \\
\hline
\end{tabular}

Results are expressed as mean $\pm \mathrm{SD}(n=14$ for synbiotic group and, $n=15$ for placebo group). $p>0.05$. Note. The prefeeding period was week 1 , the feeding period was week 6 , and the postfeeding period was week 8 .

Researchers proposed the hypothesis that increased intestinal polyamines levels would increase longevity in mice by improving intestinal health and inhibiting systemic chronic low-grade inflammation (Matsumoto et al., 2011). Recent results obtained with the aid of previous studies have shown that intestinal bacterial metabolites produced by Bifidobacterium animalis ssp. lactis LKM512 contributed to suppressing the inflammatory cytokine production, and that one of the antiinflammatory metabolites in the fecal extracts was likely to have been a polyamine (Kibe et al., 2014; Matsumoto \& Benno, 2006; Matsumoto et al., 2011). Contrary to these results, the present findings indicated that the consumption of synbiotic or placebo beverages has not resulted in significant changes IL- 6 as well as TNF- $\alpha$, the typically produced proinflammatory cytokines in the murine macrophage-like cell line RW364 (Table 3). In addition, IL-10 levels have not changed significantly in any of the two groups during the study periods. Consistent with a previous study and despite the differences in methodologies, the authors focused on the fecal Bifidobacterium microbiota composition and serum cytokines of institutionalized elderly subjects, either consuming a fermented oat drink containing two probiotic strains, a product containing a commercial B. animalis ssp. lactis BB-12, or a placebo product without probiotic bacteria, and reported that the serum levels of IL-10 and TNF$\alpha$ have not changed significantly in any of the treatment groups during the study periods (Ouwehand et al., 2008). However, they also concluded that the serum cytokines levels correlated with the presence and the levels of specific Bifidobacterium strains, which may provide a means of influencing the inflammatory responses in elderly individuals. In fact, the literature has already been demonstrated the capacity of probiotics to improve the immune system and health (Moura et al., 2016; Lollo, Morato, Moura, Almada et al., 2015; Jain, Gupta, \& Jain, 2014; Nagpal et al., 2012; Lollo et al., 2013).

There is substantial evidence suggesting that polyamines assist in healthy aging. However, further research is required before making recommendations on optimal and safe polyamine intake. This is because demonstrating unequivocal cause and effect of polyamines in healthy or disease prevention conditions requires the determination of polyamine content within the tissues under consideration, and human tissue samples are usually not readily available (Hunter \& Burrit, 2012). Furthermore, as with other tumors, colorectal cancer (CRC) is known to be linked to an increase in polyamine content if compared to with the adjacent mucosa and equivalent normal tissues, thus suggesting that this increase is due to the loss of polyamine homeostasis, which occurs during the dysregulation of cell proliferation (Linsalata \& Russo, 2008). However, even nowadays, it has been reported that gut microorganisms may promote CRC onset and progression by different process, such as (1) the induction of chronic inflammatory state; (2) the biosynthesis of genotoxins; (3) the production of toxic metabolites; and (4) the activation of dietary heterocyclic amines (HCAs) and pro-carcinogenic 
compounds (Candela et al., 2014).

At present, it has been proposed that maintaining endogenous polyamine concentrations with advanced age in the absence of tumor tissues has a positive influence on sustaining good health conditions (Matsumoto et al., 2009). Moreover, for instance, an approach for CRC prevention surely relies on probiotic bacteria usage, such as Bifidobacterium and Lactobacillus (Candela et al., 2014). The precise mechanism by which probiotic bacteria influence polyamine biosynthesis and, more generally, cell proliferation are not sufficiently clear, but it is likely that these effects may proceed through diverse metabolic and physiological modifications (Linsalata et al., 2005).

\section{Conclusions}

In the current clinical trial it has been demonstrated that synbiotic product consumption taking in daily doses by elderly people does not significantly influence the fecal microbiota. However, the consumption of synbiotic or placebo beverages, containing functional ingredients (soy and yacon as natural prebiotic source), besides their inherent nutritional value, increased polyamines levels, which are often reduced in the elderly population, without influencing inflammatory responses. Whether this may improve intestinal health is yet to be investigated, which is a suggestion for further studies.

\section{Acknowledgements}

The authors would like to thank Fundação de Amparo a Pesquisa do Estado de São Paulo (FAPESP) (Process number: 07/55331-0) for the financial support and fellowship and Christian Hansen for supplying of the probiotic culture (BB-12 ${ }^{\circledR}$-Probiotic-culture-Probio-Tec ${ }^{\circledR}$ ).

\section{References}

Alander, M., Matto, J., Kneifel, W., Johansson, M., Kogler, M., Crittenden, R., ... Saarelaa, M. (2001). Effect of galacto-oligosaccharide supplementation on human faecal microflora and on survival and persistence of Bifidobacterium lactis Bb-12 in the gastrointestinal tract. International Dairy Journal, 11, 817-825.

Bartosch, S., Fite, A., Macfarlane, G. T., \& McMurdo, M. E. T. (2004). Characterization of bacterial communities in feces from healthy elderly volunteers and hospitalized elderly patients by using real-time PCR and effects of antibiotic treatment on the fecal microbiota. Applied and Environmental Microbiology, 70(6), 3575-3581.

Bartosch, S., Woodmansey, E. J., Paterson, J. C. M., McMurdo, M. E., \& Macfarlane, G. T. (2005). Microbiological effects of consuming a synbiotic containing Bifidobacterium bifidum, Bifidobacterium lactis, and oligofructose in elderly people, determined by real-time polymerase chain reaction and counting of viable bacteria. Clinical Infectious Diseases, 40(1), 28-37.

Biagi, E., Candela, M., Fairweather-Tait, S., Franceschi, C., \& Brigidi, P. (2012). Ageing of the human metaorganism: The microbial counterpart. Age, 34, 247-267.

Biagi, E., Nylund, L., Candela, M., Ostan, R., Bucci, L., Pini, E., ... De Vos, W. (2010). Through ageing, and beyond: Gut microbiota and inflammatory status in seniors and centenarians. PloS One, 5(5), e10667. http://dx.doi.org/10.1371/journal.pone. 0010667.

Binh, P. N. T., Soda, K., \& Kawakami, M. (2011). Mediterranean diet and polyamine intake: Possible contribution of increased polyamine intake to inhibition of age-associated disease. Nutrition and Dietary Supplements, 3, 1-7.

Candela, M., Turroni, S., Biagi, E., Carbonero, F., Rampelli, S., Fiorentini, C., \& Brigidi, P. (2014). Inflammation and colorectal cancer, when microbiota-host mutualism breaks. World Journal of Gastroenterology, 20(4), 908-922.

Cirilo, M. P. G., Coelho, A. F. S., Araujo, C. M., Gonçalves, F. R. B., Nogueira, F. D., \& Gloria, M. B. A. (2003). Profile and levels of bioactive amines in green and roasted coffee. Food Chemistry, 82, 397-402.

Claesson, M. J., Cusacka, S., O'Sullivan, O., Greene-Diniz, R., De Weerdd, H., Flannery, E., .. O'Toole, P. W. (2011). Composition, variability, and temporal stability of the intestinal microbiota of the elderly. Proceedings of the National Academy of Sciences, 108(1), 4586-4591.

Glória, M. B. A., Tavares-Neto, J., \& Labanca, R. A. (2005). Influence of cultivar and germination on bioactives amines in soybeans (Glycine max L. Merril). Journal of Agricultural and Food Chemistry, 53(19), 7480-7485.

Hunter, D. C., \& Burrit, D. J. (2012). Polyamines of plant origin - An important dietary consideration for human health, phytochemicals as nutraceuticals - Global approaches to their role in nutrition and health. ISBN: 978-953-51-0203-8 In V. Rao (Ed.), InTech. Retrieved from: http://www.intechopen.com/books/phytochemicals as-nutraceuticals-global-approaches-to-their-role-innutrition-and-health/ polyamines-of-plant-origin-an-important-dietary-consideration-for-human-health.

Jain, M., Gupta, k., \& Jain, P. (2014). Significance of probiotics and prebiotics in healthy and nutrition. Malaya Journal of Biosciences, 1(3), 181-195.

Kantaria, U. D., \& Gokani, R. H. (2011). Quality and safety of biogenic amines.
International Journal of Research in Pharmaceutical and Biomedical Sciences, 2(4), 1461-1468.

Kibe, R., Kurihara, S., Sakai, Y., Suzuki, H., Ooga, T., Sawaki, E., ... Matsumoto, M. (2014). Upregulation of colonic luminal polyamines produced by intestinal microbiota delays senescence in mice. Scientific Reports, 4, 4548. http://dx.doi.org/10. 1038/srep04548.

Lahtinen, S. J., Tammela, L., Korpela, J., Parhiala, R., Ahokoski, H., Mykkänen, H., \& Salminen, S. J. (2009). Probiotics modulate the Bifidobacterium microbiota of elderly nursing home residents. Age, 31, 59-66.

Linsalata, M., \& Russo, F. (2008). Nutritional factors and polyamine metabolism in colorectal cancer. Nutrition, 24(4), 382-389.

Linsalata, M., Russo, F., Berloco, P., Valentini, A. M., Caruso, M. L., Simone, C., ... Di Leo, A. (2005). Effects of probiotic bacteria (VSL\#3) on the polyamine biosynthesis and cell proliferation of normal colonic mucosa of rats. In Vivo, 19, 989-996.

Lollo, P. C. B., Morato, P. N., Moura, C. S., Almada, C. N., Felicio, T. L., Esmerino, E. A., Cruz, A. G. (2015a). Hypertension parameters are attenuated by the continuous consumption of probiotic Minas cheese. Food Research International, 76, 611-617.

Lollo, P. C. B., Morato, P. N., Moura, C. S., Oliveira, M. M., Cruz, A. G., Faria, J. A. F., Cristianini, M. (2015b). Ultra-high temperature plus dynamic high pressure processing: An effective combination for potential probiotic fermented milk processing which attenuate exercise-induced immune suppression in Wistar rats. Journal of Functional Foods, 14, 541-548.

Lollo, P. C. B., Moura, C. S., Morato, P. N., Cruz, A. G., Castro, W. F., Betim, C. B., Amaya-Farfan, J. (2013). Probiotic yogurt offers higher immune-protection than probiotic whey beverage. Food Research International, 54, 118-124.

Manzoni, M. S. J., Cavallini, D. C. U., Pauly-Silveira, N. D., Roselino, M. N., \& Rossi, E. A. (2012). Development of a synbiotic low-calorie beverage made from soy and yacon extracts. Food and Nutrition Sciences, 3, 1500-1508.

Matsumoto, M., \& Benno, Y. (2004). Consumption of Bifidobacterium lactis LKM512 yogurt reduces gut mutagenicity by increasing gut polyamine contents in healthy adult subjects. Mutation Research, 568(2), 147-153.

Matsumoto, M., \& Benno, Y. (2006). Anti-inflammatory metabolite production in the gut from consumption of probiotic yogurt containing Bifidobacterium animalis subsp. lactis LKM512. Bioscience, Biotechnology and Biochemistry, 70(6), 1287-1292.

Matsumoto, M., Kurihara, S., Kibe, R., Ashida, H., \& Benno, Y. (2011). Longevity in mice is promoted by probiotic-induced suppression of colonic senescence dependent on upregulation of gut bacterial polyamine production. PloS One, 6(8), e23652. http:// dx.doi.org/10.1371/journal.pone.0023652.

Matsumoto, M., Ohishi, H., \& Benno, Y. (2001). Impact of LKM512 yogurt on improvement of intestinal environment of the elderly. FEMS Immunolology and Medical Microbiology, 31, 181-186.

Matsumoto, M., Sakamoto, M., \& Benno, Y. (2009). Dynamics of fecal microbiota in hospitalized elderly fed probiotic LKM512 yogurt. Microbiology and Immunology, 53, 421-432. http://dx.doi.org/10.1111/j.1348-0421.2009.00140.x.

Mohan, R., Koebnick, C., Schildt, J., Sschimidt, S., Mueller, M., Possner, M., ... Blaut, M. (2006). Effects of Bifidobacterium lactis Bb12 supplementation on intestinal microbiota of preterm infants: A double-blind, placebo-controlled, randomized study. Journal of Clinical Microbiology, 44(11), 4025-4031.

Moura, C.S., Lollo, P.C.B., Morato, P.N., Esmerino, E.A., Margalho, L.P., Santos-Junior,V. A., Coimbra, P. T., Cappato, L.P., Silva, M.C., Garcia-Gomes, A.S., Granato, D., Bolini, H.M. A., Sant'Ana, A.S., Cruz, A.G., Amaya-Farfan, J. 2016. Assessment of antioxidant activity, lipid profile, general biochemical and immune system responses of Wistar rats fed with dairy dessert containing Lactobacillus acidophilus La-5. Food Research International, 90, 275-280.

Munoa, F. J., \& Pares, R. (1988). Selective medium for isolation and enumeration of Bifidobacterium spp. Applied Environmental Microbiology, 54(7), 1715-1718.

Nagpal, R., Kumar, A., Kumar, M., Behare, P. V., Jain, S., \& Yadav, H. (2012). Probiotics, their health benefits and applications for developing healthier foods: A review. FEMS Microbiology Letters, 334, 1-15.

Noack, J., Dongowski, G., Hartmann, L., \& Blaut, M. (2000). The human gut bacteria Bacteroides thetaiotaomicron and Fusobacterium varium produce putrescine and spermidine in cecum of pectin-fed gnotobiotic rats. Journal of Nutrition, 130, 1225-1231.

Noack, J., Kleessen, B., Proll, J., Dongowaki, G., \& Blaut, M. (1998). Dietary guar gum and pectin stimulate intestinal microbial polyamine synthesis in rats. Journal of Nutrition, 128, 1385-1391.

Ouwehand, A. C., Bergsma, N., Parhiala, R., Lahtinen, S., Gueimonde, M., Finne-Soveri, H., ... Salminen, S. (2008). Bifidobacterium microbiota and parameters of immune function in elderly subjects. FEMS Immunology and Medical Microbiology, 53, 18-25.

Ouwehand, A. C., Tiihonen, K., Saarinen, M., Puttala, H., \& Rautonen, N. (2009). Influence of a combination of Lactobacillus acidophilus NCFM and lactitol on healthy elderly: Intestinal and immune parameters. British Journal of Nutrition, 101, 367-375.

Palaria, A., Johnson-Kanda, I., \& O'Sullivan, D. J. (2011). Effect of a synbiotic yogurt on levels of fecal Bifidobacteria, Clostridia, and Enterobacteria. Applied and Environmental Microbiology, 78(4), 933-940. http://dx.doi.org/10.1128/AEM 05848-11.

Reyed, M. R. (2007). The role of Bifidobacteria in health. Research Journal of Medical Sciences, 2(1), 14-24.

Roberfroid, M. B. (1998). Prebiotics and synbiotic: Concepts and nutritional properties. British Journal of Nutrition, 80, S197-S202.

Roberfroid, M. B. (2005). Introducing inulin-type fructans. British Journal of Nutrition, 93(1), S13-S25.

Sabater-Molina, M., Larqué, E., Torrella, F., Plaza, J., Ramis, G., \& Zamora, S. (2011). Effects of fructooligosaccharides on cecum polyamine concentration and gut maturation in early-weaned piglets. Journal of Clinical Biochemistry and Nutrition, 48(3), 230-236.

Soda, K., Kano, Y., Sakuragi, M., Takao, K., Lefor, A., \& Konishi, F. (2009). Long-term oral polyamine intake increases blood polyamine concentrations. Journal of Nutritional Science and Vitaminology, 55(4), 361-366. 